\title{
Modelo para determinar acciones de calidad en la formación virtual
}

\author{
Cabero Almenara Julio \\ cabero@us.es \\ Universidad de Sevilla, Spain
}

\section{Del Prete Annachiara}

annachiara.delprete@gmail.com

Pontificia Universidad Católica de Valparaíso, Chile

\section{Arancibia Muñoz María Luisa}

marancibiam@inacap.cl

Universidad Tecnológica de Chile INACAP, Chile

\begin{abstract}
Resumen
En el presente estudio, resultado de un trabajo de investigación, se propone un modelo de ecuaciones estructurales donde se recogen algunas de las variables que podrán determinar acciones de calidad en la formación virtual. Los datos se recogieron por medio de un cuestionario a una muestra de 640 docentes de la institución superior de Chile. Entre los resultados se destaca la significación que el dominio técnico y didáctico del docente, tiene sobre su frecuencia de uso, así como en la utilización de diferentes materiales que en ella se soporta y como, todo ello en interacción, repercute en las actividades y estrategias desarrolladas.
\end{abstract}

\section{Palabras claves}

LMS, competencias digitales docentes; competencias pedagógicas; educación superior. 


\title{
Model to Determine Quality Actions in Virtual Formation
}

\author{
Cabero Almenara Julio \\ cabero@us.es \\ Universidad de Sevilla, Spain \\ Del Prete Annachiara \\ annachiara.delprete@gmail.com \\ Pontificia Universidad Católica de Valparaíso, Chile \\ Arancibia Muñoz María Luisa \\ marancibiam@inacap.cl \\ Universidad Tecnológica de Chile INACAP, Chile
}

\begin{abstract}
In the present study, the result of a research work, a model of structural equations is proposed, which includes some of the variables that can determine quality actions in virtual training. The data was collected by means of a questionnaire to a sample of 640 teachers from the superior institution in Chile. Among the results, the significance that the technical and didactic mastery of the teacher has over its frequency of use, as well as in the use of different materials that are supported and how, all in interaction, affects the activities and strategies is highlighted developed.
\end{abstract}

\section{Keywords}

LMS, digital teaching competences; pedagogical competences; higher education. 


\section{Introducción: la formación a través de plataformas virtuales}

Las plataformas de formación virtual o LMS ("Learning management system") se han convertido últimamente en los sistemas más utilizados, por las instituciones de Educación Superior, para la gestión de las acciones formativas virtuales, bien se desarrollen completamente a distancia o elearning, o se desarrollen en combinación de la enseñanza presencial o b-learning. Es por esta razón que resulta fundamental conocer cuáles son los factores que determinan y promueven una enseñanza eficaz en estos LMS, más teniendo en cuenta que en estos LMS la mayoría de actividades realizadas por los docentes en los LMS, cumplen las características de un modelo centrado más en transmitir, que en crear y construir los conocimientos y, tienden al mismo tiempo a reproducir las metodologías y a emplear los mismos recursos didácticos de una clase tradicional de tipo presencial.

Sin embargo, hoy en día ya se puede afirmar que el uso de los Ambientes Virtuales de Aprendizaje, como es la plataforma Moodle, es parte del quehacer del profesorado, (Vuopala, Hyvönen \& Järvelä, 2016; Coyago, Puente \& 2017), permite obtener buenos resultados académicos (Cabanillas, Luengo y Torres, 2019) y los estudiantes presentan buenas percepciones hacia ella (Rivero, 2018) sobretodo en el caso de las instituciones educativa que han adoptado lo LMS como sistema de gestión. Es por esta razón que resulta fundamental conocer cuáles son los factores que promueven y determinan una docencia efectiva e innovadora en espacios virtuales, sobre todo considerando que los sistemas LMS siguen desarrollando y aumentando las funcionalidades didácticas de los ambientes de aprendizaje (Del Prete y Almenara 2020; Sinclair y Aho 2018) y un fuerte interés en la investigación educativa (Moreno, 2019).

Sin duda alguna para una docencia virtual significativa y motivadora, concurren una diversidad de factores que van desde el apoyo institucional para la producción de objetos de aprendizaje para facilitar la incorporación de las TIC en la enseñanza (Cabero et al., 2010), el grado de manejo instrumental que el profesorado tenga de las herramientas, su competencias pedagógicas que le permita la aplicación de las herramientas y actividades presentes en los LMS, y su adaptación a los contenidos curriculares (Celik \& Yesilyurt, 2013; Ward y Parr, 2010) y, no por último, la frecuencia de uso que haga de la plataforma.

En el presente estudio se propone identificar algunas variables favorecedoras de crear acciones de formación virtual de calidad. Para ello se aplicará a un modelo, la técnica estadística de las ecuaciones estructurales, (Structural Equation Modeling, SEM), que permitirá analizar la robustez del modelo teórico propuesto.

Dentro de los LMS más utilizados, a nivel internacional, se destaca la plataforma Moodle, en cuanto, como señala Kerimbayev, Kultan, Abdykarimova y Akramova (2017), presenta una serie de ventajas: funciona sin modificaciones en cualquier sistema operativo compatible con PHP; funciona como un conjunto de módulos y permite agregar o eliminar elementos de forma flexible en diferentes etapas; el diseño del sitio es fácilmente operado por el conjunto de plantillas predefinidas, que podrían complementarse con los propios desarrollos; se actualiza fácilmente a las nuevas versiones. Además, la plataforma Moodle tiene un sistema interno para actualizar su propia base y restaurarla; solo requiere una única base de datos de tipo SQL y podría usarse junto con otras aplicaciones y presta especial atención a los problemas de seguridad en todas las etapas, desde la validación de los datos introducidos mediante el uso de formularios hasta las cookies de codificación. Tal es la significación que los LMS han adquirido en el proceso de enseñanza y aprendizaje que, para algunos autores, son una de las variables que nos permiten hablar de elearning 2.0 (Cabero, 2013).

Al hablar de la calidad de la formación virtual, no basta con referirnos exclusivamente a variables de carácter tecnológicos, como son la usabilidad, funcionalidad o la facilidad de manejo de las diferentes herramientas tecnológicas en ella ubicadas (Del Prete \& Almenara 2019; Marciniak y 
Gairín, 2017); sino que implica, hablar de "las interacciones complejas que se producen entre los diferentes factores (estudiante, docente, contenidos, actividades y tecnología) que intervienen en los resultados de aprendizaje, para satisfacer necesidades personales y sociales" (Ruiz y Dávila, 2016, 17).

Entre los factores determinantes para la calidad de la docencia virtual, diversos estudios (Gramp 2013; García Aretio, 2015; García, et al. 2018; Guri-Rosenblit, 2018), señalan, el rol destacado del docente por una serie de motivos: por su función en la orientación y supervisión sistemática del proceso de aprendizaje, por su acción tutorial dirigida a ayudar a los estudiantes en las dificultades de aprendizaje y por inducir la reflexión crítica sobre el proceso de enseñanza aprendizaje. Y entre la función del docente, la que ha resultado ser la más efectiva, para promover un aprendizaje auténtico en los entornos LMS y situar al estudiante en el centro del proceso de enseñanzaaprendizaje (Hsu, 2011; Silva, 2017; Rivero, 2018), ha sido su capacidad en construir e utilizar recursos didácticos, que se sirvan de una consistente combinación (Schoonenboom, 2014) entre los diferentes tipos de herramientas que brindan los LMS, y la variedad de actividades que pueden realizarse, tanto en el aula, como fuera de ella (Buchanan, Sainter \& Saunders 2013; Luo, Murray y Crompton, 2017). Como señala Silva $(2017,7-8)$ : "La formulación, diseño e implementación de eactividades son parte central del diseño instruccional online, pueden responder a diferentes finalidades como: la motivación inicial hacia la materia; las formativas orientadas a la consecución de objetivos, competencias o resultados de aprendizaje; las evaluativas, que permiten constatar el nivel de progreso de los estudiantes."

El volumen de actividades que se pueden realizar a través de las herramientas didácticas, que brindan los LMS, está solamente limitado por la creatividad y formación que el docente tenga para su aplicación, y van desde el método de proyectos de trabajo, la visita a sitios web, la elaboración de wikis, los estudios de casos, desarrollar investigaciones, análisis de casos prácticos, etc. (Cabero y Román, 2006). Actividades que, como señalan Hannon (2011), para que sean significativas, deben poseer cuatro grandes características: estar ubicadas en un contexto reconocido y comprendido por el estudiante; trasmitir el sentido didáctico y llevar al estudiante a percibir su valor práctico e intelectual; ser motivadoras, persistentes y englobadoras, para facilitar el aprendizaje constante y continuo. A tal propósito, diferentes investigaciones (Ljbojevic, Vaskovic, Stankovic y Vaskovic, 2015; Gregory y Bannister, 2017; Jenaro, Martín, Castaño y Flores, 2018) ponen de manifiesto que en la medida que los docentes incorporan en las plataformas una diversidad de materiales (documentos textuales, clip de vídeos, multimedias, podcast de audio, materiales de autoevaluación,...) fomentan y promueven la realización de una diversidad de actividades, que repercuten en crear un entorno más rico y más motivador para la adquisición de conocimientos por parte de los estudiantes, y entre todo este volumen de actividades empiezan a destacar las que favorecen el aprendizaje colaborativo (García-Chivas y Súarez-Guerrero, 2019).

Sin embargo, muchos estudios han demostrado (Blin \& Munro, 2008; Garrote, \& Pettersson, 2007; Mahdizadeh, Biemans, Mulder, 2008, Hsu, 2011) que, en la docencia virtual, la mayoría de actividades realizadas, cumplen características de un modelo centrado más en transmitir, que en crear y construir conocimientos; hecho que, desde nuestro punto de vista, viene motivado, por el tipo de formación técnica y didáctica que posea el docente, y por la frecuencia y tipo de uso que haga de la plataforma y que condiciona la realización de actividades más diversas e innovadoras (Badia, Garcia y Meneses, 2017; Luo, et al., 2017; Ramirez y Barajas, 2017).

De acuerdo con la propuesta formulada por Mishra y Koehler (2006) y Koehler y Mishra (2008) conocida como TPACK (Tecnological Pedagogical Content Knowledge), modelo que ha sido validado a través de diferentes investigaciones (Cabero y Barroso, 2016; Cejas, Navío y Barroso, 2016), para que el uso de las TIC en el aula y, en nuestro caso, de los LMS en el proceso de enseñanza y aprendizaje, sea significativo y provoque impacto en la formación de los alumnos, la capacitación de los docentes debe asegurar un sólido conocimiento de tres dimensiones, que son dimensión 
tecnológica, didáctica y pedagógica. Esta propuesta al ser analizada mediante el modelo de ecuaciones estructurales, está poniendo de manifiesto que las diferentes interacciones entre los tres conocimientos básicos, como por ejemplo entre el conocimiento tecnológico-pedagógicocontenido, establecidos por los autores, son difíciles de interpretar y comprender por los docentes, si bien la interacción conocimiento tecnológico y pedagógico si es claramente identificada (Ay, Karadağ y Acat, 2015; Pamuk, Ergun, Cakir, Yilmaz, y Ayas, 2015).

Apoyándonos en estas reflexiones, en el presente estudio se propone analizar un modelo (figura 1) donde se recogen algunas de las variables que podrían determinar acciones de calidad en la formación virtual, en particular: dominio técnico-didáctico, frecuencia de uso, diferentes tipos de materiales, actividades y estrategias desarrolladas en la plataforma.

El modelo viene a indicarnos que le dominio técnico y didáctico que le docente tenga tanto de la plataforma en general, como de las diferentes herramientas particulares que la conforman, influirán tanto en la frecuencia de uso de la plataforma como en la incorporación de diferentes tipos de materiales (textuales, audiovisuales, multimedia y de autoevaluación), repercutiendo todos ellos en la realización de diferentes actividades y estrategias en la formación virtual realizada por los docentes.

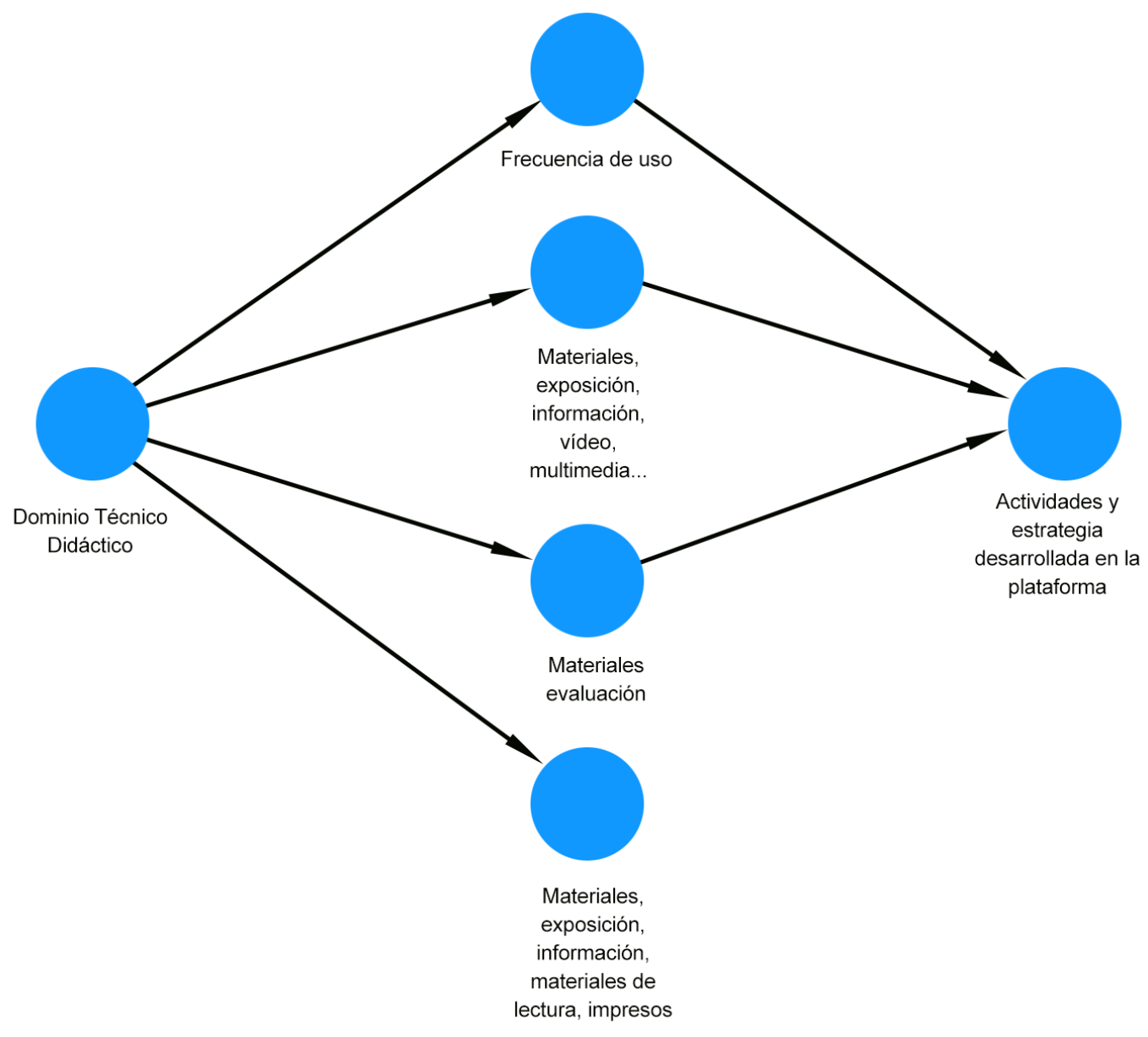

Fuente: elaboración propia Ilustración 1 Modelo Elaborado 
El modelo propuesto será analizado mediante ecuaciones estructurales, para verificar su significación global y la significación de cada una de las variables sobre el modelo.

\section{Metodología}

Los objetivos perseguidos en el estudio son: a) Validar mediante el modelo de ecuaciones estructurales (Structural Equation Modeling, SEM), la viabilidad del modelo propuesto, b) Analizar la relación entre las diversas variables propuestas en el modelo.

\section{a. Muestra}

La muestra estuvo formada por 640 docentes de la institución INACAP de Chile de todas sus sedes y de todas las carreras impartidas en la institución. Los datos se recogieron durante el curso académico 2017-18. El muestreo utilizado es de tipo no probabilístico (Alaminos, 2006; Sabariego, 2012), que se caracteriza por la facilidad de acceso por parte del investigador a los sujetos que la forman. De los 640, 231 (36,09\%) eran hombres y 409 (63,91\%) mujeres.

\section{b. Diseño de la investigación}

El diseño utilizado en la investigación es del tipo "expo-facto" que son aquellos en los cuales el investigador no manipula ninguna variable, sino que analiza el fenómeno cuando ya ha ocurrido y que no han sido provocadas por el investigador (Mateo, 2004; Albert, 2007).

\section{c. Instrumentos de recogida de información}

El instrumento de recogida de información estaba compuesto por 2 grandes ítems con construcción tipo Likert, en uno se le preguntaba al docente que puntuara de 1 (muy poco) a 10 (muchísimo), el dominio técnico, el manejo didáctico y la frecuencia de utilización que realizaba de diferentes herramientas incorporadas en la plataforma Moodle: foros, blog, wikis, tareas, etc. $Y$ en otro, que puntuara el grado de utilización de la plataforma Moodle para el desarrollo de diferentes actividades y estrategias didácticas que podía realizar con la plataforma (seguimiento del trabajo académico del alumno, realización de actividades colaborativas, formulación de problemas, etc.). El instrumento se administró vía web.

\section{Resultados}

Indicar desde el principio que los SEM (Structural Equation Modeling), o modelos de ecuaciones estructurales SEM, son modelos estadísticos multivariantes, que facilitan conocer el efecto y las relaciones entre una diversidad de las variables. Respecto a los modelos de regresión Son menos restrictivos (Ruiz, Pardo y San Martín, 2010, 34); siendo su gran potencialidad la de poder representar el efecto causal entre las variables de un modelo. Son una ampliación de la regresión múltiple y el análisis factorial (Byrne, 2013). Desde esta perspectiva, una de sus principales aportaciones es que facilitan evaluar y analizar los modelos teóricos formulados (Cabero y Pérez, 2018); siendo su campo de acción: servir para la confirmación de modelos teóricos establecidos, para contrastar modelos teóricos rivales, y para el desarrollo de un modelo específico (Cupani, 2012), y estimar y evaluar la relación variable latentes que son constructos no observables, e indicadores que son las diferentes variables a través de las cuales pueden medirse las variables latentes. 
En el presente trabajo para su realización utilizamos los programas SmartPLS y Adanco.

Su aplicación supone seguir una serie de pasos: obtención del índice de fiabilidad, obtención de las cargas, hallazgos de la fiabilidad compuesta, elaboración de las varianzas extraídas, medias, que son los pasos que a continuación vamos a analizar para la validación del modelo que se ha elaborado.

En primer lugar, se obtuvo el índice de fiabilidad, mediante la alfa de Cronback, que es el estadístico usual para instrumentos con construcción tipo Likert (O'Dwyer y Bernauer, 2014), Los valores obtenidos se presentan en la tabla $n^{\circ} 1$.

\begin{tabular}{|l|c|}
\hline & Alfa Cronback \\
\hline Dominio técnico y didáctico & 0,96 \\
\hline Frecuencia uso & 0,89 \\
\hline Actividades & 0,90 \\
\hline Materiales lectura & 0,79 \\
\hline Materiales audiovisuales y multimedia & 0,78 \\
\hline Materiales evaluación & 0,70 \\
\hline
\end{tabular}

Tabla 1: Alfa de Cronbach de las diferentes variables latentes consideradas

Como se puede observar todas las dimensiones que hemos establecido superan los valores del 0,7 , o se ubican en él, a partir de los cuales se consideran niveles adecuados de fiabilidad ( $O$ 'Dwyer y Bernauer, 2014), lo cual nos permite señalar desde el comienzo los altos niveles de fiabilidad.

El siguiente paso fue obtener las cargas, o correlaciones simples, de los indicadores con su respectivo constructo, que se presentan en la tabla $n^{0} 2$, debemos señalar que para que un indicador se considere que forma parte del constructo, debe poseer una carga superior a 0,707 (Carmines y Zeller, 1979). Hecho que se da en la gran mayoría de los casos.

\begin{tabular}{|c|c|c|c|c|c|c|}
\hline Indicator & DTD & $\overline{F U}$ & A & M1 & $\overline{\mathrm{M} 2}$ & $\mathrm{M} 3$ \\
\hline D. Técnico Foro & 0,7571 & & & & & \\
\hline D. Técnico Blog & 0,7777 & & & & & \\
\hline D. Técnico Wiki & 0,7775 & & & & & \\
\hline D. Técnico Tareas & 0,6393 & & & & & \\
\hline D. Técnico Glosario & 0,7482 & & & & & \\
\hline D. Técnico Vídeos & 0,6651 & & & & & \\
\hline D. Técnico Video/Audioconferencia & 0,7666 & & & & & \\
\hline D. Técnico Cuestionario on-line & 0,7986 & & & & & \\
\hline D. Técnico Co-evaluaciones & 0,7749 & & & & & \\
\hline D. Técnico Consultas o encuestas & 0,7687 & & & & & \\
\hline M. Didáctico Foro & 0,7385 & & & & & \\
\hline
\end{tabular}



M. Didáctico Blog
0,7982
M. Didáctico Wiki
0,7914
M. Didáctico Tareas
0,5900
M. Didáctico Glosario
0,7492
M. Didáctico Vídeos
0,6467
M. Didáctico Vídeo/Audioconferencia
0,7736
M. Didáctico Cuestionarios on-line
0,8034
M. Didáctico Co-evaluaciones
0,7476
M. Didáctico Consultas o encuestas
0,7597

F. Uso Foro

0,7108

F. Uso Blog

0,7559

F. Uso Wiki

0,7680

F. Uso Tareas

0,5104

F. Uso Glosario

0,8045

F. Uso Vídeos

0,6118

F. Uso Video/Audioconferencia

0,7262

F. Uso Cuestionario on-line

0,7298

F. Uso Co-evaluaicones

0,7721

F. Uso Consultas o encuestas

0,7099

A. Retroalimentar el trabajo académico del alumno

A. Realizar actividades de trabajo colaborativo

0,6650

A Plantear y proponer problemas

0,6604

A Trabajar con proyectos

0,6874

A Trabajar con la metodología de estudios de casos

A. Realizar debates o foros de discusión

A Usar simulaciones

A. Entregar premios, puntuaciones, ranking

0,7259

A. Impulsar reflexión en los estudiantes a través de diferentes herramientas

A. Utilizar herramientas para que los alumnos debatan

A. Utilizar herramientas para que los alumnos realizaran ensayos

0,7509

M. Guía de estudio 
M. Guías de taller o laboratorio

M. Apuntes

M. Presentaciones en power-point

M. Vídeos

M. Archivos de audio

M. Animaciones, fotografías,...

M. Multimedia interactivo

M. Ejercicios de autoevaluación

M. Exámenes on-line

M. Simuladores - laboratorio virtual

M. Artículos de lectura (pdf)

M. Libros electrónicos

M Manuales
0,6006

0,5792

0,6074

0,7714

0,7527

0,7782

0,8078

0,8170

0,7891

0,7557

Tabla 2: Cargas. (Nota: DTD=Dominio técnico y didáctico; FU=Frecuencia de uso; A=Actividades; M1=Materiales lectura; $\mathrm{H} 2=$ Materiales audiovisuales y multimedia; $\mathrm{H} 3=$ Materiales de evaluación)

A continuación, se obtuvo el análisis de la "fiabilidad compuesta" mediante la rho de Jöreskog's (Cho, 2016). De nuevo es la puntuación de 0,7 la que establece el nivel mínimo correcto. En la tabla no 3 pueden observarse las puntuaciones obtenidas, que superan el valor crítico indicado.

\begin{tabular}{|l|c|}
\hline & Jöreskog's rho $\left(\boldsymbol{\rho}_{\mathbf{c}}\right)$ \\
\hline Dominio técnico y didáctico & 0,9614 \\
\hline Frecuencia uso & 0,9115 \\
\hline Actividades & 0,9180 \\
\hline Materiales lectura & 0,8455 \\
\hline Materiales audiovisuales y multimedia & 0,8596 \\
\hline Materiales evaluación & 0,8305 \\
\hline
\end{tabular}

Tabla 3: Fiabilidad compuesta

Seguidamente se obtuvo la validez convergente, que persigue estudiar si un conjunto de indicadores representa un único constructo subyacente o varios, y que se valora mediante la "Varianza Extraída Media" (AVE). En este caso los valores deben ser superiores al 0,5 para que más del $50 \%$ de las varianzas del constructo sea debida a los indicadores (Baggozi y Yi, 1988). Los valores alcanzados se ofrecen en la tabla no 4, y nos muestra que, salvo en un caso, se confirman la validez convergente. 


\begin{tabular}{l|c}
\hline \hline Constructo & AVE \\
\hline Dominio técnico y didáctico & 0,5563 \\
\cline { 1 - 1 } Frecuencia uso & 0,5109 \\
\cline { 1 - 1 } & 0,5063 \\
\cline { 1 - 1 } & 0,4419 \\
\hline Matividades & 0,6049 \\
\hline Materiales audiovisuales y multimedia & 0,6204 \\
\hline
\end{tabular}

Tabla 4: Varianzas extraídas media.

Posteriormente se logró la validez discriminante, que requiere la obtención de dos tipos de análisis: el criterio de Fornell-Larcker (tabla $n^{\circ} 5$ ) y las cargas factoriales cruzadas (tabla $n^{\circ} 6$ ). El primero, se apoya en que la varianza media extraída ha de ser mayor que la varianza que dicho constructo comparte con los otros constructos del modelo; de manera similar, las correlaciones entre los constructos han de ser menores, que la raíz cuadrada de la varianza media extraída. En la tabla no 7 los elementos de la diagonal son la raíz cuadrada de la varianza extraída media y los de fuera de la diagonal son las correlaciones entre constructos. Para que se cumpla el requisito, los valores que estén a la izquierda y por debajo de cada valor en negrita, han de ser menores que él valor arriba indicado, hecho cumplido en todos los casos.

\begin{tabular}{|c|c|c|c|c|c|c|}
\hline Constructo & DTD & $\overline{F U}$ & $\bar{A}$ & M1 & M2 & M3 \\
\hline Dominio técnico y didáctico & 0,5563 & & & & & \\
\hline Frecuencia uso & 0,3849 & 0,5109 & & & & \\
\hline Actividades & 0,2306 & 0,4944 & 0,5063 & & & \\
\hline Materiales lectura & 0,1570 & 0,1517 & 0,3022 & 0,4419 & & \\
\hline Materiales audiovisuales y multimedia & 0,1370 & 0,2743 & 0,3407 & 0,2213 & 0,6049 & \\
\hline Materiales evaluación & 0,1302 & 0,3445 & 0,4062 & 0,1829 & 0,3488 & 0,6204 \\
\hline
\end{tabular}

Tabla 5: Criterio de Fornell-Larcker (Nota: DTD=Dominio técnico y didáctico; FU=Frecuencia de uso; A=Actividades; M1=Materiales lectura; H2=Materiales audiovisuales y multimedia; H3=Materiales de evaluación)

Posteriormente se obtuvo el análisis de las cargas cruzadas, que pretende estudiar si el ítem de un constructo mide lo que constructo persigue medir y no mide aspectos de otro constructo; para que tales ítems sean significativos deben cargar más fuertemente sobre su propio constructo que sobre otros. Aspecto que como podemos observar en la tabla $n^{0} 6$ se cumple en nuestro caso cumple en nuestro caso como se observa en la tabla $n^{\circ} 6$. 


\begin{tabular}{|c|c|c|c|c|c|c|}
\hline Indicador & DTD & $\mathrm{FU}$ & $\mathrm{A}$ & M1 & M2 & M3 \\
\hline D. Técnico Foro & 0,7571 & 0,4094 & 0,3510 & 0,3237 & 0,2290 & 0,2303 \\
\hline D. Técnico Blog & 0,7777 & 0,3554 & 0,3063 & 0,2558 & 0,1907 & 0,1897 \\
\hline D. Técnico Wiki & 0,7775 & 0,3948 & 0,3282 & 0,2929 & 0,2258 & 0,2102 \\
\hline D. Técnico Tareas & 0,6393 & 0,3758 & 0,4191 & 0,4359 & 0,3061 & 0,2185 \\
\hline D. Técnico Glosario & 0,7482 & 0,4212 & 0,3464 & 0,2603 & 0,2802 & 0,2149 \\
\hline D. Técnico Vídeos & 0,6651 & 0,3563 & 0,3215 & 0,3624 & 0,3963 & 0,2060 \\
\hline D. Técnico Video/Audioconferencia & 0,7666 & 0,4045 & 0,3230 & 0,2854 & 0,2704 & 0,3059 \\
\hline D. Técnico Cuestionario on-line & 0,7986 & 0,4399 & 0,3140 & 0,2993 & 0,2428 & 0,3650 \\
\hline D. Técnico Co-evaluaciones & 0,7749 & 0,4787 & 0,3421 & 0,2111 & 0,1710 & 0,2610 \\
\hline D. Técnico Consultas o encuestas & 0,7687 & 0,4878 & 0,3551 & 0,2954 & 0,2839 & 0,3007 \\
\hline M. Didáctico Foro & 0,7385 & 0,5312 & 0,4062 & 0,3109 & 0,2771 & 0,2539 \\
\hline M. Didáctico Blog & 0,7982 & 0,4992 & 0,3535 & 0,2315 & 0,2126 & 0,2641 \\
\hline M. Didáctico Wiki & 0,7914 & 0,5045 & 0,3588 & 0,2602 & 0,2021 & 0,2642 \\
\hline M. Didáctico Tareas & 0,6900 & 0,4079 & 0,4008 & 0,4050 & 0,2745 & 0,2279 \\
\hline M. Didáctico Glosario & 0,7492 & 0,5432 & 0,4017 & 0,2324 & 0,3199 & 0,2360 \\
\hline M. Didáctico Vídeos & 0,6467 & 0,4298 & 0,3500 & 0,3731 & 0,4339 & 0,2455 \\
\hline M. Didáctico Vídeo/Audioconferencia & 0,7736 & 0,5127 & 0,3657 & 0,2845 & 0,3347 & 0,3445 \\
\hline M. Didáctico Cuestionarios on-line & 0,8034 & 0,5042 & 0,3226 & 0,2717 & 0,2433 & 0,3758 \\
\hline M. Didáctico Co-evaluaciones & 0,7476 & 0,5292 & 0,3551 & 0,1957 & 0,2243 & 0,2737 \\
\hline M. Didáctico Consultas o encuestas & 0,7597 & 0,5588 & 0,3861 & 0,2760 & 0,3085 & 0,3149 \\
\hline F. Uso Foro & 0,4414 & 0,7108 & 0,5715 & 0,2714 & 0,3619 & 0,3809 \\
\hline F. Uso Blog & 0,4651 & 0,7559 & 0,4903 & 0,2474 & 0,3355 & 0,4416 \\
\hline F. Uso Wiki & 0,4528 & 0,7680 & 0,5309 & 0,2573 & 0,3411 & 0,4591 \\
\hline F. Uso Tareas & 0,3637 & 0,6104 & 0,4709 & 0,3361 & 0,3154 & 0,2638 \\
\hline F. Uso Glosario & 0,4563 & 0,8045 & 0,5732 & 0,2842 & 0,4717 & 0,4252 \\
\hline F. Uso Vídeos & 0,4201 & 0,6118 & 0,4540 & 0,3628 & 0,5105 & 0,3096 \\
\hline F. Uso Video/Audioconferencia & 0,4611 & 0,7262 & 0,4854 & 0,2828 & 0,3985 & 0,4613 \\
\hline F. Uso Cuestionario on-line & 0,5031 & 0,7298 & 0,4476 & 0,2729 & 0,3344 & 0,5367 \\
\hline F. Uso Co-evaluaciones & 0,4550 & 0,7721 & 0,5112 & 0,2450 & 0,3288 & 0,4551 \\
\hline F. Uso Consultas o encuestas & 0,3957 & 0,7099 & 0,4629 & 0,2329 & 0,3386 & 0,4345 \\
\hline $\begin{array}{l}\text { A. Retroalimentar el trabajo académico del } \\
\text { alumno }\end{array}$ & 0,3432 & 0,4410 & 0,6172 & 0,3336 & 0,3574 & 0,3551 \\
\hline $\begin{array}{l}\text { A. Realizar actividades de trabajo } \\
\text { colaborativo }\end{array}$ & 0,3357 & 0,4768 & 0,6650 & 0,3857 & 0,3706 & 0,3388 \\
\hline A Plantear y proponer problemas & 0,3037 & 0,4045 & 0,6604 & 0,4485 & 0,3228 & 0,3542 \\
\hline
\end{tabular}


A Trabajar con proyectos

A Trabajar con la metodología de estudios de casos

A. Realizar debates o foros de discusión

A Usar simulaciones

A. Entregar premios, puntuaciones, ranking

A. Impulsar reflexión en los estudiantes a través de diferentes herramientas

A. Utilizar herramientas para que los alumnos debatan

A. Utilizar herramientas para que los alumnos realizaran ensayos

M. Guía de estudio

M. Guías de taller o laboratorio

M. Apuntes

M. Presentaciones en power-point

M. Vídeos

M. Archivos de audio

M. Animaciones, fotografías,...

M. Multimedia interactivo

M. Ejercicios de autoevaluación

M. Exámenes on-line

M. Simuladores - laboratorio virtual

M. Artículos de lectura (pdf)

M. Libros electrónicos

M Manuales

\begin{tabular}{|c|c|c|c|c|c|}
\hline 0,3366 & 0,4473 & 0,6874 & 0,4920 & 0,3250 & 0,3864 \\
\hline 0,2763 & 0,4369 & 0,6823 & 0,5156 & 0,3131 & 0,3917 \\
\hline 0,3175 & 0,5239 & 0,7469 & 0,3607 & 0,3904 & 0,4509 \\
\hline 0,2353 & 0,4333 & 0,6535 & 0,3085 & 0,4045 & 0,5372 \\
\hline 0,2935 & 0,5364 & 0,7259 & 0,2859 & 0,4198 & 0,5623 \\
\hline 0,4662 & 0,5888 & 0,7885 & 0,4311 & 0,5001 & 0,5245 \\
\hline 0,4024 & 0,6075 & 0,8216 & 0,3739 & 0,5424 & 0,5550 \\
\hline 0,4168 & 0,5466 & 0,7509 & 0,4405 & 0,5326 & 0,4499 \\
\hline 0,1902 & 0,1741 & 0,2101 & 0,6017 & 0,2351 & 0,2042 \\
\hline 0,2025 & 0,1813 & 0,2828 & 0,6006 & 0,2863 & 0,3074 \\
\hline 0,1679 & 0,1479 & 0,1746 & 0,6192 & 0,1601 & 0,1401 \\
\hline 0,2599 & 0,2183 & 0,3321 & 0,6074 & 0,3065 & 0,1808 \\
\hline 0,3858 & 0,4142 & 0,4458 & 0,4545 & 0,7714 & 0,3070 \\
\hline 0,1797 & 0,4115 & 0,4871 & 0,2734 & 0,7527 & 0,5904 \\
\hline 0,2319 & 0,3507 & 0,4118 & 0,3806 & 0,7782 & 0,4722 \\
\hline 0,3308 & 0,4443 & 0,4686 & 0,3464 & 0,8078 & 0,4887 \\
\hline 0,3191 & 0,5352 & 0,5754 & 0,3616 & 0,4854 & 0,8170 \\
\hline 0,2803 & 0,4526 & 0,4722 & 0,3111 & 0,4375 & 0,7891 \\
\hline 0,2461 & 0,3827 & 0,4440 & 0,3359 & 0,4736 & 0,7557 \\
\hline 0,2955 & 0,3627 & 0,4955 & 0,7465 & 0,3978 & 0,3492 \\
\hline 0,3453 & 0,3050 & 0,4584 & 0,7362 & 0,3417 & 0,3654 \\
\hline 0,3088 & 0,3332 & 0,4575 & 0,7528 & 0,3855 & 0,3613 \\
\hline
\end{tabular}

Tabla 6: Matriz de cargas cruzadas. (Nota: DTD=Dominio técnico y didáctico; FU=Frecuencia de uso; $A=$ Actividades; $M 1=$ Materiales lectura; $H 2=$ Materiales audiovisuales y multimedia; H3=Materiales de evaluación)

Posteriormente pasamos a estudiar los coeficientes de regresión estandarizados ("path coefficients"), los valores de la t de student y los R2 (R-cuadrado); coeficiente que determina el porcentaje de varianza de un constructo que es explicado por las variables predictoras del mismo. En la figura no 2, se presentan los valores obtenidos. 


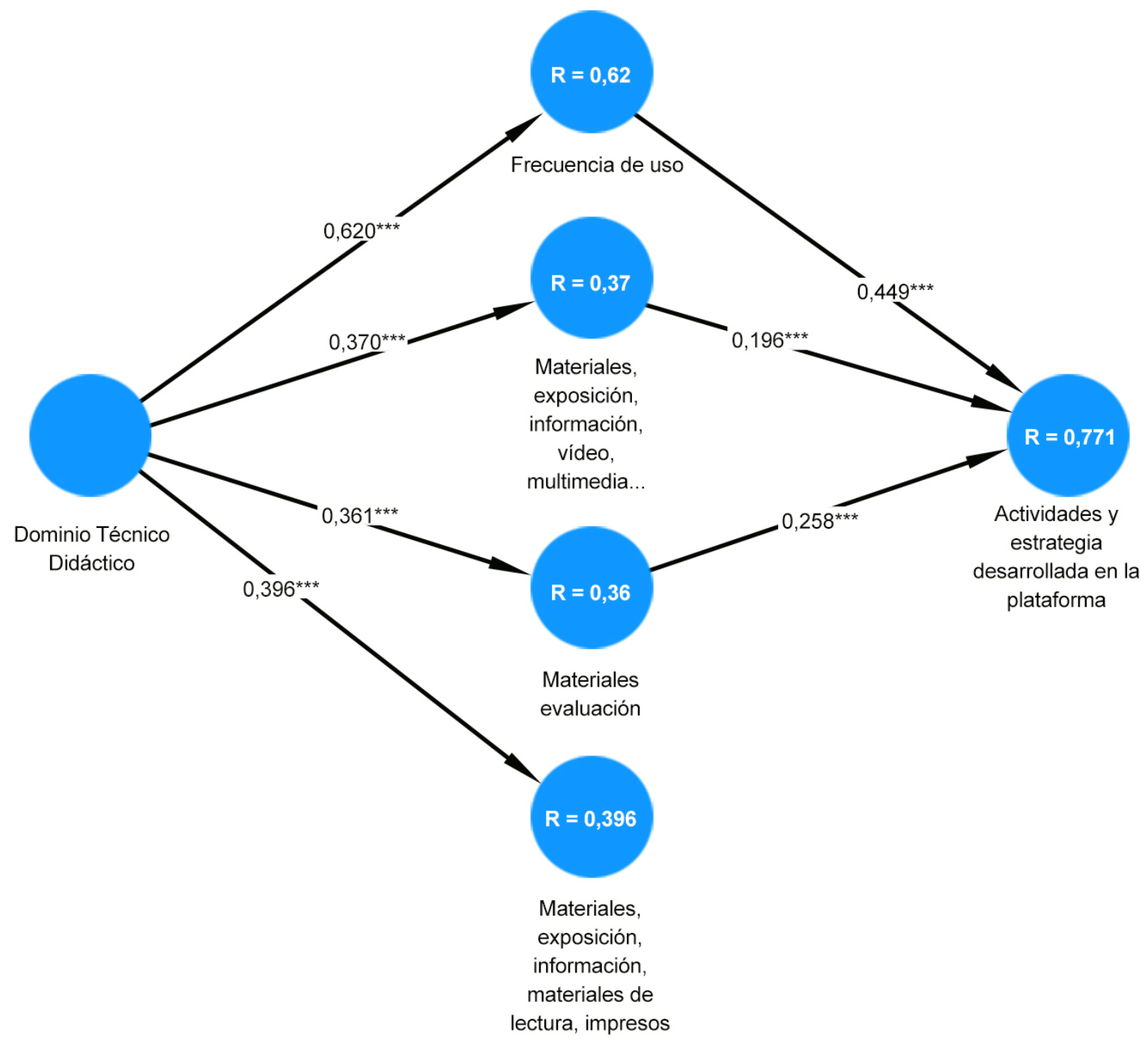

Fuente: elaboración propia

llustración 2: Valores R2 y niveles de significación de las variables.

Respecto a las variables latentes del modelo formulado, y tras estudio llevado a cabo, hallamos que el $13,7 \%$ de la varianza de la variable latente "Materiales impresos" es explicada por las variables latentes "Dominio técnico-didáctico"; el 38,5\% de la varianza de la variable latente "Frecuencia de uso" está explicada por las variables latentes "Dominio técnico-didáctico"; el 13,0\% de la varianza de la variable latente, "Materiales de evaluación" está explicado por las variables latentes "Dominio técnico-didáctico"; el 15,7\% de la varianza de la variable latente "Materiales impresos" está explicado por las variables latentes "Dominio técnico-didáctico", y el 59,5\% de la varianza de la variable "Actividades y estrategias desarrolladas en la plataforma" está explicado por la variable latente "Materiales audiovisuales y multimedia", "Materiales de evaluación" y "Frecuencia de utilización."

En la tabla $n^{0} 7$ se ofrece el porcentaje de varianza explicado de las variables latentes por sus determinadas variables productoras. 


\begin{tabular}{|c|c|c|c|c|}
\hline Variable dependiente & Variables predictoras & Path & Correlación & $\begin{array}{c}\text { Varianza } \\
\text { explicada }\end{array}$ \\
\hline Frecuencia uso & Dominio técnico - didáctico & 0,6204 & 0,6204 & $38,5 \%$ \\
\hline Materiales lectura & Dominio técnico - didáctico & 0,3962 & 0,3962 & $15,7 \%$ \\
\hline Materiales audiovisuales & Dominio técnico - didáctico & 0,3702 & 0,3702 & $13,7 \%$ \\
\hline Materiales evaluación & Dominio técnico - didáctico & 0,3609 & 0,3609 & $13,0 \%$ \\
\hline \multirow{2}{*}{ Actividades } & Frecuencia uso & 0,4489 & 0,7031 & $31,6 \%$ \\
\cline { 2 - 5 } & Materiales audiovisuales & 0,1962 & 0,5837 & $11,5 \%$ \\
\cline { 2 - 5 } & Materiales de evaluación & 0,258 & 0,6374 & $16,4 \%$ \\
\hline
\end{tabular}

Tabla 7: Varianza explicada por cada variable latente.

También es importante analizar si los valores obtenidos son significativos, aplicando para ello el estadístico t de student de los valores "path", utilizando la técnica de bootstrap. En la tabla no 8 se ofrecen los valores obtenidos.

\begin{tabular}{|c|c|c|c|c|c|}
\hline \multicolumn{6}{|c|}{ Valores t y p-valores } \\
\hline & $\begin{array}{c}\text { Muestra } \\
\text { original }\end{array}$ & $\begin{array}{c}\text { Media } \\
\text { muestra }\end{array}$ & $\begin{array}{c}\text { Desviación } \\
\text { estándar }\end{array}$ & $\begin{array}{c}\text { Estadísticos } \\
\mathbf{t}\end{array}$ & P Valores \\
\hline D. técnico-didáctico -> F. uso & 0,6204 & 0,6207 & 0,0550 & 11,2769 & $0,0000^{* *}$ \\
\hline D. técnico-didáctico -> M. lectura & 0,3962 & 0,4112 & 0,0622 & 6,3669 & $0,0000^{* *}$ \\
\hline D. técnico-didáctico -> M. audiovisuales & 0,3702 & 0,3737 & 0,0620 & 5,9663 & $0,0000^{* *}$ \\
\hline D. técnico-didáctico -> M. de evaluación & 0,3609 & 0,3625 & 0,0649 & 5,5602 & $0,0000^{* *}$ \\
\hline F. uso $->$ Actividades & 0,4489 & 0,4572 & 0,0821 & 5,4686 & $0,0000^{* *}$ \\
\hline M. audiovisuales -> Actividades & 0,1962 & 0,1948 & 0,0643 & 3,0487 & $0,0024^{* *}$ \\
\hline M. de evaluación -> Actividades & 0,2580 & 0,2584 & 0,0715 & 3,6106 & $0,0003^{* *}$ \\
\hline
\end{tabular}

Tabla 8: Valores t de Student y p-valores de los coeficientes path (nota: ${ }^{* *}=$ significativo al 0,01

Los valores alcanzados nos llevan a señalar que todas las relaciones establecidas son significativas al nivel de significación 0,01 o inferior.

El modelo también indica la influencia de los diferentes indicadores en las variables latentes analizadas. Y de nuevo se observa, en la tabla no 2 ubicada anteriormente, que los valores de las cargas son bastante altos y superan en todos los casos el valor 0,60. En concretos los ítems formulados para la variable latente "Dominio técnico y didáctico", las cargas de los ítems van desde 0,6393 (dominio técnico del vídeo) a 0,8034 (Dominio didáctico cuestionario on-line); en los formulados para la "frecuencia de uso" desde 0,6104 (frecuencia de uso tareas) a 0,8045 (frecuencia de uso glosario), en "Actividades y estrategias" desde 0,6172 (retroalimentar el trabajo académico de los alumno) a 0,8216 (utilizar herramientas para que los alumnos debatan), en "Materiales de lectura" desde 0,6017 (guía de estudio) a 0,6192 (apuntes), en "Materiales audiovisuales y multimedia" con 0,7527 (animaciones, fotografías,...) y 0,8078 (multimedia 
interactivo) y por lo que se refiere a los "Materiales de evaluación" con 0,7557 (simuladores laboratorios virtuales) y 0,8170 (ejercicios de autoevaluación).

Finalmente obtuvimos el indicador SRMR (Standardized Root Mean square, que nos es de utilidad para evaluar la bondad de ajuste del modelo estructural se utilizó), alcanzándose una puntuación del 0,077, que, al ser menor de 0,08, nos sugiere un buen ajuste del modelo (Hu y Bentler, 1999).

\section{Discusión de resultados y conclusiones}

Nuestra primera conclusión va a ir dirigida a la validez y fortaleza de la técnica estadística SEM para analizar y validar modelos, y no exclusivamente para alcanzar el índice de fiabilidad, sino fundamentalmente para estudiar la significación del modelo elaborado, la influencia que cada ítem presenta sobre el constructo en el cual se ha insertado, la duplicidad de influencias de ítems sobre los constructos, y la significación del constructo completo. En definitiva, nos ha servido para evaluar la potencia de modelos teóricos formulados. En el caso concreto el modelo construido hace referencia a la significación que el dominio técnico y didáctico del docente, respecto a la plataforma Moodle, tiene sobre su frecuencia de uso, así como en la utilización de diferentes materiales que en ella se soportan y como, todo ello en interacción, repercute en las actividades y estrategias desarrolladas a través de la citada plataforma de formación virtual.

Respecto a la fiabilidad del instrumento elaborado para el examen de las diferentes dimensiones que hemos incorporado en el proyecto, tenemos que decir que son elevadas tanto desde la perspectiva de la alfa de Cronbach como por la fiabilidad compuesta analizada a través del estadístico rho de Jöreskog.

Independientemente de la visión general del modelo, el SEM ha puesto de manifiesto que el "dominio técnico y didáctico" que el docente tenga de la plataforma Moodle influye directa y significativamente en la frecuencia de utilización que haga de la misma. Frecuencia de utilización que influye significativamente en la diversidad de actividades y estrategias que el docente es capaz de movilizar con la plataforma Moodle. Por otra parte, el dominio técnico y didáctico que el docente indica poseer de la plataforma Moodle, repercute directa y significativamente sobre la utilización que hace de los materiales de lectura, audiovisuales y multimedia, y de evaluación.

Ha sido relevante el hecho de no haber encontrado relaciones significativas entre los materiales de lectura y la realización de actividades y estrategias desarrolladas en la plataforma; por el contrario, si se obtuvieron entre los materiales audiovisuales y multimedia, y de evaluación. Dicho de otro modo, los docentes utilizan los materiales de lectura (guías de estudio, guías de taller o laboratorio, artículos de lectura, apuntes, manuales,...) para transmitir información, y los audiovisuales y multimedia (vídeos, archivos de audio, animaciones,...), y de evaluación (ejercicios de autoevaluación, exámenes on-line, ...) para la realización de actividades con los estudiantes; ellos coincide con lo expuesto por Samaniego, Marqués y Gisbert (2015) que llaman la atención respecto a la tendencia de los docentes a contemplar las plataformas, más que como impulsoras del desarrollo de actividades, como transmisoras de información.

Lo obtenido nos lleva a señalar, por una parte, la necesidad de una formación del profesorado que supere la simple capacitación tecnológica y alcance la dimensión pedagógica y educativa, planteamiento que ha sido defendido por diferentes autores y a través de distintos modelos, como el del TPACK (Mishra y Koehler, 2006). Como han apuntado recientemente Samperio y Barragán (2018) al señalar que, aun reconociendo las ventajas que ofrecen las plataformas para la enseñanza, para que los alumnos accedan a materiales y realicen diferentes actividades: 
no es suficiente incluir la plataforma en el proceso educativo; es necesaria una adecuada capacitación que permita obtener los conocimientos tecnológicos, pedagógicos y de contenido que ayuden al usuario a adquirir el potencial que esta herramienta tecnológica puede aportar en el aprendizaje de los alumnos (p. 129).

Es necesario insistir en la necesidad de que el docente incorpore materiales diferentes a los textuales, y que son los que determinan la calidad del entorno (Ljbojevic, et al., 2015; Gregory y Bannister, 2017; Jenaro, et al., 2018). De todas formas, creemos que es necesario capacitar a los docentes para que adquieran competencias para desarrollar actividades a partir de los materiales de lectura. Por otra parte, se confirma lo expresado por diferentes autores (Badia, et al., 2017; Luo, et al., 2017; Ramírez y Barajas, 2018) respecto a que un aumento de uso de la plataforma, conlleva un aumento de actividades por parte del docente. Como ha señalado Guri-Rosenblit (2018), los estudiantes percibe con claridad cuando sus docentes no se encuentran preparados para la desarrollar su actividad profesional en contextos virtuales, lo que los lleva a desentenderse y despreocuparse por la formación. De ahí la necesidad de, por parte de las instituciones, se establezcan las medidas técnicas y organizativas para facilitar su utilización por los docentes.

\section{Limitaciones e investigaciones futuras}

Entre las limitaciones que presenta el estudio señalamos que la muestra de estudio, fue recogida en una única universidad y sería interesante ampliar el análisis a otras instituciones, para verificar la tendencia señalada por el modelo presentado. Junto con esto se señala que el instrumento utilizado es de tipo autoinformes, por ello pensamos que se hace necesario la utilización de otros, como pudieran ser la observación no participante de las acciones realizadas con TIC, la realización de entrevistas en profundidad, y el análisis de "big data", de las acciones realizadas tanto por profesores como profesoras en la plataforma virtual. Los datos recogidos con estos instrumentos facilitarían su triangulación con los obtenidos en nuestro estudio, y consolidar y apuntalar los hallazgos.

Por último, teniendo en cuenta la utilidad de los análisis SEM, para la validación del modelo, como investigaciones futuras se sugiere construir otros modelos a partir de otras dimensiones, que puedan influir en la utilización de la plataforma Moodle por los docentes, como por ejemplo las creencias que los docentes tienen sobre modalidades diferentes de enseñanza, el grado de aceptación que los docentes tienen sobre la tecnología Moodle, analizado mediante el modelo TAM formulado en su momento por Davis (1989), o la significación que sobre el modelo pueden tener determinadas variables como pueden ser el género del docente o la disciplina en la cual desarrolla su actividad profesional de la enseñanza.

\section{Referencias}

Alaminos, A. (2006). El muestreo en la investigación social. En A. Alaminos y J.L. Castejón (Eds.), Elaboración, análisis e interpretación de encuestas, cuestionarios y escalas de opinión (pp. 46-67). Alcoy: Marfil.

Albert, M.J. (2007). La investigación educative. Madrid: McGraw-Hill.

Arbuckle, J. L. (1997). Amos Users' Guide: Version 3.6. Chicago: SmallWaters Corporation.

Ay, Y., Karadağ, E. y Acat, M. (2015). The Technological Pedagogical Content Knowledge-practical (TPACKPractical) model: Examination of its validity in the Turkish culture via structural 
equation modeling. Computers \& Education, 88, 97-108. DOI: $10.1016 /$ j.compedu.2015.04.017

Badia, A., Garcia, C. y Meneses, J. (2017). Approaches to teaching online: Exploring factors influencing teachers in a fully online university. British Journal of Educational Technology, 48(6), 1193-1207. DOI:10.1111/bjet.12475.

Bagozzi, R. P., \& Yi, Y. (1988). On the evaluation of structural equation models. Journal of the academy of marketing science, 16(1), 74-94; DOI:10.1007/BF02723327.

Blin, F., \& Munro, M. (2008). Why hasn't technology disrupted academics' teaching practices? Understanding resistance to change through the lens of activity theory. Computers \& Education, 50(2), 475-490. DOI:10.1016/j.compedu.2007.09.017

Byrne, B. M. (2013). Structural equation modeling with EQS: Basic concepts, applications, and programming. Routledge.

Browne, T., Hewitt, R., Jenkins, M., \& Walker, R. (2008). 2008 survey of technology enhanced learning for higher education in the UK. Oxford, UK: Universities and Colleges Information Systems Association

Buchanan, T., Sainter, P. \& Saunders, G. (2013). Factors affecting faculty use of learning technologies: implications for models of technology adoption. Journal of Computing in Higher Education, 25(1), 1-11. DOI: 10.1007/s12528-013-9066-6

Cabanillas, J.L., Luengo, R. y Torres, J.L. (2019). Diferencias de actitud hacia las TIC en la formación profesional en entornos presenciales y virtuales (Plan @vanza). Pixel-Bit. Revista de Medios y Educación, 55, 37-55, DOI: https://doi.org/10.12795/pixelbit.2019.i55.03

Cabero, J., Morales, J. A., Barroso, J. M., Fernández, J. M., Romero, R., Román, P. \& Ballesteros, C. (2010). Análisis de centros de recursos de producción de las TIC de las universidades españolas. Revista de Educación, 351, 237-257. Recuperado de: https://core.ac.uk/download/pdf/51386907.pdf

Cabero, J. (2013). La formación virtual en el nuevo entramado 2.0: el e-learning 2.0. Tecnologías y medios para la educación en la e-sociedad, 19, 23-51.

Cabero, J. y Barroso, J. (2016). ICT teacher training: a view of the TPACK model / Formación del profesorado en TIC: una visión del modelo TPACK, Cultura y Educación, 28(3), 633663. Recuperado de: http://dx.doi.org/10.1080/11356405.2016.1203526

Cabero, J. y Pérez Díez, J.L. (2018). Validación del modelo TAM de adopción de la Realidad Aumentada mediante ecuaciones estructurales. Estudios sobre Educación, 34, 129-153. DOI: $10.15581 / 004.34 .129-153$

Cabero, J. y Román, P (2006). E-actividades: un referente básico para la formación en Internet. MAD-Eduforma.

Carmines, E. G. \& Zeller, R. A. (1979). Reliability and validity assessment (Vol. 17). Sage publications.

Celik, V. \& Yesilyurt, E. (2013). Attitudes to technology, perceived computer self-efficacy and computer anxiety as predictors of computer supported education. Computers \& Education, 60(1), 148-158. Recuperado de: https://www.sciencedirect.com/science/article/pii/S0360131512001509

Cejas, R., Navío, A. y Barroso, J. (2016). Las competencias del profesorado universitario desde el modelo TPACK (conocimiento tecnológico y pedagógico del contenido). Pixel-Bit. Revista de 
Medios $y$ Educación, 49, 105-119. Recuperado de: https://recyt.fecyt.es/index.php/pixel/article/viewFile/61717/37727

Cho, E. (2016). Making Reliability Reliable: A Systematic Approach to Reliability Coefficients. Organizational Research Methods, 19(4), 651-682. DOI: 10.1177/1094428116656239

Coyago, A. P. R., Puente, E. \& Jiménez, G. A. M. (2017). Uso de las tecnologías de la información en la educación superior. INNOVA Research Journal, 2(1), 99-112 Recuperado de: https://dialnet.unirioja.es/servlet/articulo?codigo $=5920546$

Cupani, M. (2012). Análisis de Ecuaciones Estructurales: conceptos, etapas de desarrollo y un ejemplo de aplicación. Revista Tesis, (2)1, 186-199. Recuperado de : https://revistas.unc.edu.ar/index.php/tesis/article/view/2884/2750

Davis, F. (1989), Perceived usefulness, perceived ease of use, and user acceptance of information technology. MIS Quarterly, 13(3), 319-340, DOI: 10.2307/249008 Del Prete, A. \& Almenara, J. C. (2020) El uso del Ambiente Virtual de Aprendizaje entre el profesorado de educación superior: un análisis de género. RED. Revista de Educación a Distancia. 62 (20). Artíc. 04, pp1-20 DOI: http://dx.doi.org/10.6018/red.400061 Del Prete A. \& Cabero J. (2019) Las plataformas de formación virtual: algunas variables determinantes de su utilización. Apertura Revista de Innovación Educativa 11(2) E-ISSN 2007-1094. http://dx.doi.org/10.32870/ap.v11n2.1521.

Garrote, R., \& Pettersson, T. (2007). Lecturers' attitudes about the use of learning management systems in engineering education: a Swedish case study. Australasian Journal of Educational Technology, 23, 327-349. Recuperado de: https://ajet.org.au/index.php/AJET/article/view/1256

García, B., Serrano, E. L., Ponce, S., Cisneros-Cohernour, E. J., Cordero, G., y Espinosa, Y. (2018). Las competencias docentes en entornos virtuales: un modelo para su evaluación. RIED. Revista Iberoamericana de Educación a Distancia, 21(1), 343-365. DOI: $10.5944 /$ ried.21.1.18816

García Aretio, L. (2015). MOOC: ¿tsunami, revolución o moda pasajera?. RIED. Revista Iberoamericana de Educación a Distancia, 18(1), 9-21. Recuperado de: http://revistas.uned.es/index.php/ried/article/view/13812

García-Chitiva, M. P. y Suárez-Guerrero, C. (2019). Estado de la investigación sobre la colaboración en Entornos Virtuales de Aprendizaje. Pixel-Bit. Revista de Medios y Educación, 56, 169-191. https://doi.org/10.12795/pixelbit.2019.i56.09

Gramp, J. (2013, October). Beyond the baseline: working with e-learning champions to transform e-learning at a research-led university. Second Moodle Research Conference, 24-32.

Gregory, S. y Bannister, M. (2017). Digital learner presence and online teaching tools: higher cognitive requirements of online learners for effective learning. Technology Enhanced Learning, 12(18), 1-17. DOI 10.1186/s41039-017-0059-3

Guri-Rosenblit, S. (2018). E-Teaching in Higher Education: An Essential Prerequisite for E-Learning. Journal of New Approaches in Educational Research, 7(2), 93-97. doi: 10.7821/naer.2018.7.298

Hannon, V. (2011). Learning Futures. A contribution to the Innovative Learning Environments project of OECD-CERI. Recuperado de: http://www.oecd.org/edu/ceri/Valerie\%20Hannon.Learning\%20Futures.pdf. 
Hsu, S. (2011). Who Assigns the Most ICT Activities? Examining the Relationship between Teacher and Student Usage. Computers \& Education 56(3): 847-855. Recuperado de: https://www.sciencedirect.com/science/article/pii/S0360131510003180

Hu, L. y Bentler, P. (1999). Cutoff criteria for fit indexes in covariance structure analysis: Conventional criteria versus new alternatives. Structural Equation Modeling: A Multidisciplinary Journal, 6(1), 1-55. DOI:10.1080/10705519909540118

Jenaro, C., Martín, M.E., Castaño, R. y Flores, N. (2018). Rendimiento académico en educación superior y su asociación con la participación activa en la plataforma Moodle. Estudios sobre Educación, 34, 177-198. DOI: 10.15581/004.34.177-198

Kahn, J. H. (2006). Factor analysis in Counseling Psychology research, training and practice: Principies, advances and applications. The Counseling Psychologist, 34, 1-36. DOI $10.1177 / 0011000006286347$

Kerimbayev, N., Kultan, J. Abdykarimova, S. y Akramova, A. (2017). LMS Moodle: Distance international education in cooperation of higher education institutions of different countries. Educ Inf Technol, 22(5), 2125-2139. DOI: 10.1007/s10639-016-9534-5

Kerlinger, F. y Lee, H. (2002). Investigación del comportamiento. Métodos de investigación en las ciencias sociales. México: McGraw-Hill.

Koehler, J., y Mishra, P. (2008). Technological pedagogical content knowledge (TPACK) the development and validation of an assessment instrument for preservice teachers. Journal of Research on Technology in Education, 42(2), 123-149. Recuperado de: https://files.eric.ed.gov/fulltext/EJ868626.pdf

Ljbojevic, M., Vaskovic, V., Stankovic, S. y Vaskovic, J. (2015). El uso del vídeo complementario en la enseñanza multimedia como herramienta didáctica para incrementar la eficiencia del aprendizaje y la calidad de experiencia. Revista Mexicana de Bachillerato a Distancia, (7)13, 134-153. Recuperado

de: http://www.revistas.unam.mx/index.php/rmbd/article/view/64999

Luo, T., Murray, A. y Crompton, H. (2017). Designing Authentic Learning Activities to Train PreService Teachers About Teaching Online. International Review of Research in Open and Distributed Learning, 18(7), 141-157. Recuperado de: http://www.irrodl.org/index.php/irrodl/article/view/3037/4440

Mahdizadeh, H., Biemans, H. \& Mulder, M. (2008). Determining factors of the use of e-learning environments by university teachers. Computers \& Education, 51(1), 142-154. DOI: 10.1016/j.compedu.2007.04.004

Mateo, J. (2004). La investigación ex post-facto. En R., Bisquerra (Coord.), Metodología de la investigación cualitativa (pp. 195-230). Madrid: La Muralla.

Marciniak, R. y Gairín, J. (2017). Un modelo para la autoevaluación de la calidad de programas de educación universitaria virtual. RED. Revista de Educación a Distancia, 54(2), 1-30. DOI: $10.6018 / \mathrm{red} / 54 / 2$

Mishra, P. y Koehler, M. (2006). Technological pedagogical content knowledge: A new framework for teacher knowledge. Teachers College Record, 108(6), 1017- 1054. Recuperado de: http://one2oneheights.pbworks.com/f/MISHRA_PUNYA.pdf

Moreno, J. (2019). Estudio bibliométrico de la producción científica en Web of Science: Formación Profesional y blended learning. Pixel-Bit. Revista de Medios y Educación, 56, 149-168. https://doi.org/10.12795/pixelbit.2019.i56.08. 
O'Dwyer, L. y Bernauer, J. (2014). Quantitative research for the qualitative researcher. California: Sage.

Pamuk, S., Ergun, M., Cakir, R., Yilmaz, y Ayas, C. (2015). Exploring relationships among TPACK components and development of the TPACK instrument. Educational Informational Technology, 20(2), 241-263. DOI: 10.1007/s10639-013-9278-4

Ramirez, W. y Barajas, J.I. (2017). Uso de las plataformas educativas y su impacto en la práctica pedagógica en instituciones de educación superior de San Luis Potosí. EDUTEC, Revista Electrónica de Tecnología Educativa, 60. Recuperado de: http://edutec.es/revista/index.php/edutec-e/article/view/798

Rivero, M. (2018). Percepción estudiantil sobre la calidad de un ambiente de aprendizaje mixto apoyado por Moodle. Pixel-Bit. Revista de Medios y Educación, 53, 193-205, doi: http://dx.doi.org/10.12795/pixelbit.2018.i53.13

Ruiz, C. y Dávila, A. A. (2016). Propuesta de buenas prácticas de educación virtual en el contexto universitario. RED-Revista de Educación a Distancia, 49(12). DOI: 10.6018/red/49/12

Ruiz, M., Pardo, A. y San Martín, R. (2010). Modelo de ecuaciones estructurales. Papeles del psicólogo, 31(1), 34-45. Recuperado de: http://www.papelesdelpsicologo.es/pdf/1794.pdf

Samaniego, G., Marqués, L., y Gisbert, M. (2015). El profesorado universitario y el uso de Entornos Virtuales de aprendizaje. Campus Virtuales, 4(2), 50-58. Recuperado de: http://uajournals.com/ojs/index.php/campusvirtuales/article/view/84

Sabariego, M. (2012). El proceso de investigación (parte 2). En Bisquerra, R. (coord.), Metodología de la investigación educativa (pp. 127-163). (3a ed.). Madrid: La Muralla.

Samperio, V. y Barragán, J. (2018). Análisis de la percepción de docentes, usuarios de una plataforma educativa a través de los modelos TPACK, SAMR y TAM3 en una institución de educación superior. Apertura, 10(1), 116-131. DOI: 10.18381/ap.v10n1.1162

Schoonenboom, J. (2014). Using an adapted, task-level technology acceptance model to explain why instructors in higher education intend to use some learning management system tools more than others. Computers \& Education, 71, 247-256. Recuperado de: https://www.sciencedirect.com/science/article/pii/S0360131513002790

Silva, J. (2017). Un modelo pedagógico virtual centrado en las E-actividades. RED. Revista de Educación a Distancia, 53(10), 1-20. DOI: 10.6018/red/53/10

Sinclair, J. \& Aho, A. M. (2018). Experts on super innovators: understanding staff adoption of learning management systems. Higher Education Research \& Development, 37(1), 158172. DOI: $10.1080 / 07294360.2017 .1342609$

Vuopala, E., Hyvönen, P. y Järvelä, S. (2016). Interaction forms in successful collaborative learning in virtual learning environments. Active Learning in Higher Education,17(1), 25-38. DOI: $10.1177 / 1469787415616730$

Ward, L. and Parr, J.M. (2010). Revisiting and reframing use: implications for the integration of ICT. Computers and Education, 54(1), 113-122. Recuperado de: https://www.sciencedirect.com/science/article/pii/S0360131509001882 